\title{
ON A THEOREM BY I. GLICKSBERG
}

A. E. NUSSBAUM ${ }^{1}$

If $X$ is a compact space we denote by $C(X)$ the Banach space of all continuous complex valued functions on $X$ with respect to the norm $\|f\|=\sup _{x \in X}|f(x)|$. Grothendieck [2, Theorem 5] has shown that a bounded subset of $C(X)$ is compact in the weak topology if and only if it is compact in the topology of pointwise convergence on $X$.

Using an extension of this theorem I. Glicksberg [1, Theorem 1.2] recently proved

Theorem 1. Let $X$ and $Y$ be locally compact Hausdorff spaces, and $f$ a bounded complex function on $X \times Y$ which is separately continuous, i.e., for which all the maps

$$
x \rightarrow f(x, y) \text { and } y \rightarrow f(x, y)
$$

are continuous. Then for $\mu$ a bounded Radon measure on $Y$,

$$
x \rightarrow \int f(x, y) d \mu(y)
$$

is continuous.

The purpose of this note is to prove the following more general

THEOREM 2. Let $X$ be a locally compact and $Y$ a locally compact Hausdorff space. Let $f$ be a complex function on $X \times Y$ which is separately continuous, and $\mu$ a Radon measure on $Y$. Suppose furthermore that $|f(x, y)| \leqq g(y)$ for all $x \in X$ and almost all $y \in Y$ (i.e., for all $y \in Y$ except a $\mu$-null set), where $g \in L^{1}(\mu)$. Then

$$
x \rightarrow \int f(x, y) d \mu(y)
$$

is continuous.

Proof. The proof could be derived from Theorem 1. However, we prefer to derive it directly from Grothendieck's theorem by a slight modification of Glicksberg's proof.

Let $\epsilon$ be any positive number. Since $g$ is zero outside the union of a $\mu$-null set and a countable set of compact sets, and every compact set

Received by the editors June 29, 1961.

${ }^{1}$ Research supported by a grant from the National Science Foundation under contract NSF-G17932. 
$K \subset Y$ contains a compact set $K_{1}$ such that $\mu\left(K-K_{1}\right)$ is arbitrarily small and the restriction of $g$ to $K_{1}$ is continuous, there exists a compact set $K \subset Y$ such that the restriction of $g$ to $K$ is continuous, and such that

$$
\int_{Y-K} g(y) d \mu(y)<\epsilon .
$$

We may furthermore assume that $K$ is such that

$$
|f(x, y)| \leqq g(y) \text { for all } x \in X \text { and all } y \in K .
$$

Since $g$ is continuous on $K$, there exists a constant $M$ such that $|f(x, y)| \leqq M$ for all $x \in X$ and all $y \in K$.

Let now $a$ be any point of $X$ and $\left(x_{\alpha}\right)$ be any generalized sequence (i.e., directed set) of points in $X$ which converges to $a$, and $V$ be a compact neighborhood of $a$. Denote by $f_{x}$ the restriction of the mapping $y \rightarrow f(x, y)$ to $K$. Then $A=\left\{f_{x}\right\}, x \in V$, is a bounded subset of $C(K)$ which is compact in the topology of pointwise convergence on $X$, since the mapping $x \rightarrow f_{x}$ of $X$ into $C(K)$ endowed with the topology of pointwise convergence on $K$ is continuous. Hence by Grothendieck's theorem $A$ is compact in the weak topology of $C(K)$ and the two topologies agree on $A$ since they are comparable. It follows that $\left(f_{x_{\alpha}}\right), x_{\alpha} \in V$, converges weakly to $f_{x}$. Hence there exists an $\alpha$ such that

$$
\left|\int_{K}\left[f(a, y)-f\left(x_{\beta}, y\right)\right] d \mu(y)\right|<\epsilon \quad \text { for } \beta>\alpha .
$$

Therefore

$$
\begin{aligned}
& \left|\int\left[f(a, y)-f\left(x_{\beta}, y\right)\right] d \mu(y)\right| \\
& \quad \leqq\left|\int_{K}\left[f(a, y)-f\left(x_{\beta}, y\right)\right] d \mu(y)\right|+2 \int_{Y-K} g(y) d \mu(y)<3 \epsilon \text { for } \beta>\alpha,
\end{aligned}
$$

which proves that the mapping $x \rightarrow \int f(x, y) d \mu(y)$ is continuous at $a$.

\section{REFERENCES}

1. I. Glicksberg, Weak compactness and separate continuity, Pacific J. Math. 11 (1961), 205-214.

2. A. Grothendieck, Criteres de compacite dans les espaces fonctionnels generaux, Amer. J. Math. 74 (1952), 168-186.

WASHINGTON UNIVERSITY 